\title{
Numerical Study of the Surface-Hardening Effect on Surface Phenomena in 3D Polycrystalline Specimens
}

\author{
Varvara Romanova ${ }^{1,2, a)}$, Ruslan Balokhonov ${ }^{1,2, b)}$, \\ Olga Zinovieva ${ }^{1,3}$, and Valeriy Shakhijanov ${ }^{3}$ \\ ${ }^{1}$ Institute of Strength Physics and Materials Science SB RAS, Tomsk, 634055, Russia \\ ${ }^{2}$ National Research Tomsk Polytechnic University, Tomsk, 634050, Russia \\ ${ }^{3}$ National Research Tomsk State University, Tomsk, 634050, Russia

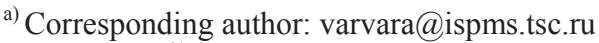 \\ b) rusy@ispms.tsc.ru
}

\begin{abstract}
Surface hardening effect on the mesoscale surface deformation in polycrystalline specimens subjected to uniaxial tension is numerically studied. Basing on the experimental findings, three-dimensional microstructure-based constitutive models of the unhardened and surface-hardened polycrystalline specimens are constructed. The mechanical behavior of the polycrystalline models is analysed numerically by the finite-difference method. The grain structure is shown to be responsible for the free surface roughening under uniaxial loading. Microscale stresses acting in the bulk of the material across the free surface give rise to the formation of surface ridges and valleys. The hardened layer in a surface-hardened specimen moves the grain structure away from the free surface, thus smoothing out the microscale folds caused by displacements of individual grains. The thicker is the modified layer, the smoother is the surface relief.
\end{abstract}

Keywords: surface hardening, surface roughening, 3D polycrystalline models, grain boundaries

\section{INTRODUCTION}

A great body of experimental and theoretical data (see, e.g., [1, 2]) indicates that plastic deformation is accompanied by the formation of a specific relief on the initially flat free surface. This phenomenon known as surface roughening is an undesirable feature deteriorating surface mechanical properties and responsible for plastic strain localization during loading. It is a challenge, therefore, to elaborate efficient methods for suppressing surface roughening mechanisms. Experiments for a number of materials (e.g., [3]) have shown that surface modification by means of strain hardening or coating deposition may effectively suppress roughening. The mechanical aspects of this effect remain to be explored, however. Our goal is to analyze these effects numerically in terms of microstructure-based simulation.

\section{EXPERIMENTAL BACKGROUNDS}

High-strength steels and titanium alloys demonstrate well-pronounced roughening under uniaxial tension [3]. The ion-beam surface irradiation provides nano-or submicrocrystalline structures in an about $6 \mu \mathrm{m}$ thick surface layer. Within the layer, the grain size gradually varies from $200-300 \mathrm{~nm}$ on the surface to 5-10 $\mu \mathrm{m}$ in the bulk of the material. Microhardness measurements suggest that the elastic modulus and yield stress of the nanocrystalline subsurface layer are about twice as high as those of the base material.

Two representative surface images and corresponding surface profiles for surface-hardened and unhardened steel specimens are shown in Fig. 1. The unhardened steel undergoes surface roughening from the outset of plastic deformation. Microscale periodic folds oriented across the tensile direction are formed on the free surface early in the plastic stage $\left(\varepsilon^{\mathrm{p}}=0.5 \%\right)$. The transverse size of the folds is about $5 \mu \mathrm{m}$ and the height is about $20 \mathrm{~nm}$.

International Conference on Physical Mesomechanics of Multilevel Systems 2014

AIP Conf. Proc. 1623, 531-534 (2014); doi: 10.1063/1.4901497

(C) 2014 AIP Publishing LLC 978-0-7354-1260-6/\$30.00 

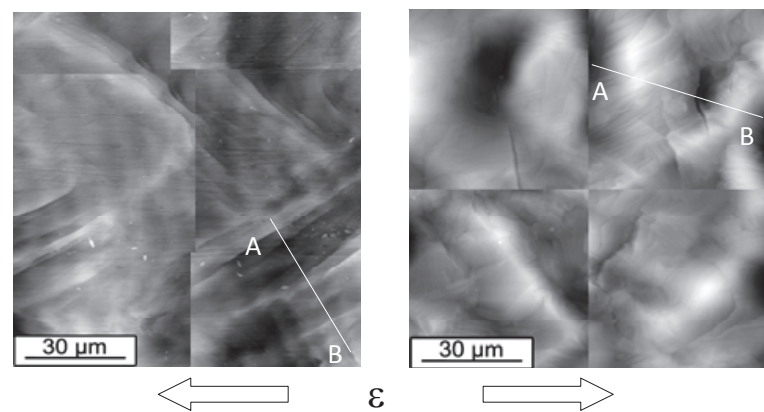

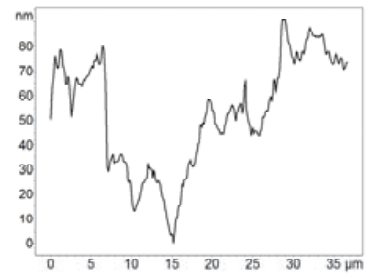

(a)

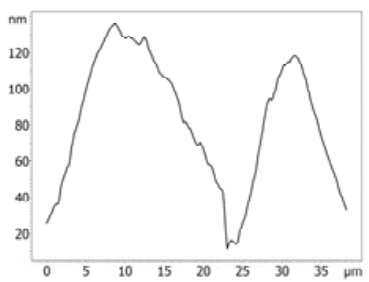

(b)

FIGURE 1. AFM images and corresponding surface profiles along $A-B$ lines of unhardened (a) and surface-hardened EK-181 steel specimens (b) under uniaxial tension, $\varepsilon=1.7 \%$ (a) and $2.7 \%$ (b)

The folds are associated with displacements of individual grains. On further loading, larger folds oriented at an angle of about $30^{\circ}$ to the tensile axis appear, with their width being approximately $2-3$ grain diameters (Fig. 1(a)), while small transverse folds continue to grow as well, forming rope-like structures. The fold width grows but slightly, while the fold height increases in a nearly linear fashion as deformation develops. A different pattern is observed in the surface-hardened specimen (Fig. 1(b)). The surface is covered by a system of relief folds whose width is equal to several grain diameters, with no small folds associated with the displacements of individual grains being observed.

\section{NUMERICAL SIMULATION}

To investigate the mechanics of surface roughening in hardened and unhardened specimens, numerical simulations were performed by means of three-dimensional models taking an explicit account of the polycrystalline structure. The polycrystalline structures have been generated by a step-by-step packing method proposed in [4]. The main idea of this method is to fill a pre-meshed volume with structural elements in accordance with a given geometrical law. In this work, the step-by-step packing procedure is applied to designing periodic polycrystalline microstructures with and without a surface-hardened layer. All grains are grown at the same rate in accordance with a spherical law until the entire computational volume is filled without gaps or overlaps. To get a periodic structure in the $X_{3}$-direction, an additional condition is imposed. If the growing grain goes beyond the surface of the computational volume, its growth continues on the opposite side by a parallel transfer.

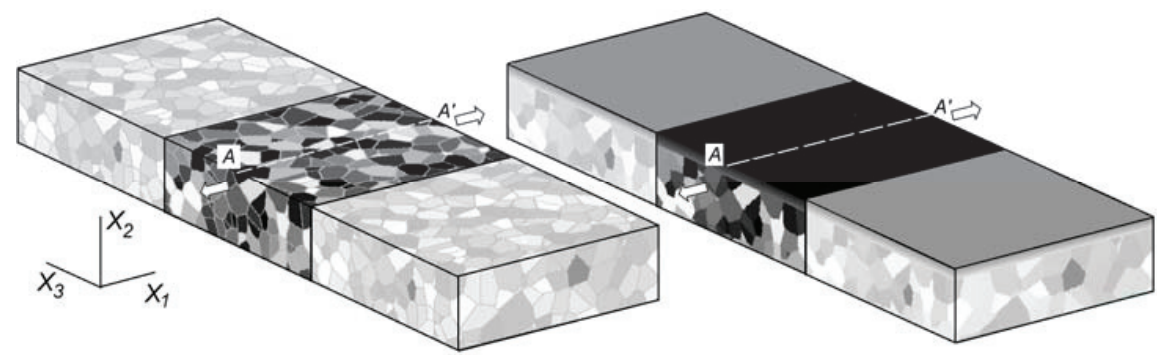

(a)

(b)

FIGURE 2. Model polycrystals without (a) and with a surface-hardened layer (b). The arrows denote the direction of tension 


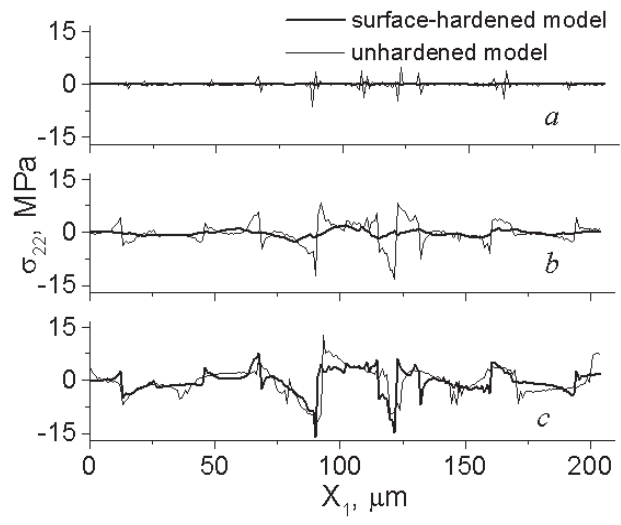

FIGURE 3. Stresses acting across the free surface in unhardened and surface-hardened specimens at a depth of 0.5 (a), 5.5 (b) and $15.5 \mu \mathrm{m}$ (c) below the surface. Strain is $2.4 \%$

Thus, we have generated a three-dimensional polycrystal consisting of 600 grains on a regular mesh of $200 \times 63 \times 200$ with a step of $1 \mu \mathrm{m}$ (Fig. 2 (a)). To design a surface-hardened polycrystal, a structureless layer has been built over the surface of the base material (Fig. 2(b)). The mechanical properties within the layer are varied linearly from the average characteristics of the base material at the internal interface to their maximum values on the surface. In the calculations, the modified surface layer thickness is varied between 5 and $15 \mu \mathrm{m}$. The constitutive model is assigned to each grain. In our calculations, an explicit definition of crystal plasticity is omitted, and the elasticplastic behavior of grains is described by a set of stress-strain curves scattered around an average curve within $20 \%$. Strain-hardening functions for particular grains were constructed to fit experimental data [3]. The average values of Young's modulus, Poisson's ratio and initial yield stress are $210 \mathrm{GPa}, 0.28$ and $264 \mathrm{MPa}$, respectively. Referring to Fig. 2, the darker is the grain, the higher is its mechanical properties.

To set boundary conditions, we introduce a system of coordinates plotted in Fig. 2(a). Tension is applied along the $X_{1}$-axis. The top surface is free from external loading. The bottom surface is a symmetry plane, its displacements being constrained in the vertical direction. Periodic boundary conditions are applied to the lateral sides.

\section{RESULTS AND DISCUSSION}

In a homogeneous material subjected to uniaxial tension, the stress tensor components normal to the axis of loading will be zero, and the free surface undergoing uniform deformation will remain flat throughout the entire loading process. In a polycrystalline material, however, all stress and strain tensor components are nonzero, including stresses acting across the free surface (Fig. 3). The sources of non-zero normal stresses are grain boundaries where the external force vector is decomposed into non-zero tangential and normal components. The non-zero normal stresses are very low in a subsurface layer and occur mainly along grain boundaries (Fig. 3(a)). At a depth of one-half the grain diameter, however, the stresses cover the entire section, demonstrating a quasi-periodic distribution of positive and negative values (Fig. 3(b)). Acting from the inside, the positive and negative stresses give rise to periodic ridges and valleys on the free surface (Fig. 4).

Based on these findings, let us compare the surface roughness in the unhardened and surface-hardened materials. Roughness patterns for the unhardened specimen and specimens with surface-hardened 5 and $15 \mu \mathrm{m}$ thick layers are presented in Fig. 4 in the form of surface images and surface profiles taken along specimen centerlines corresponding to different deformation stages. While the unhardened and surface-hardened specimens share certain common trends inherent to the surface roughness evolution, the presence of the surface-hardened layer substantially affects the roughness pattern. In all cases at hand, the roughness is in the form of quasi-periodic ridges running across the entire specimen nearly perpendicular to the tensile axis. Notably, the ridge shape changes but slightly, whereas the ridge height grows in a nearly linear fashion, as tension continues (see the surface profiles in Fig. 4). In the unhardened specimen, however, the surface roughness exhibits a well-defined experimental pattern of two sets of folds (cf. Figs. 4(a) and Fig. 1(a)). Larger folds whose transverse size is about several grain diameters are formed by grain ensembles involved in a cooperative motion. Thus the effect can be classified as mesoscale phenomena. 


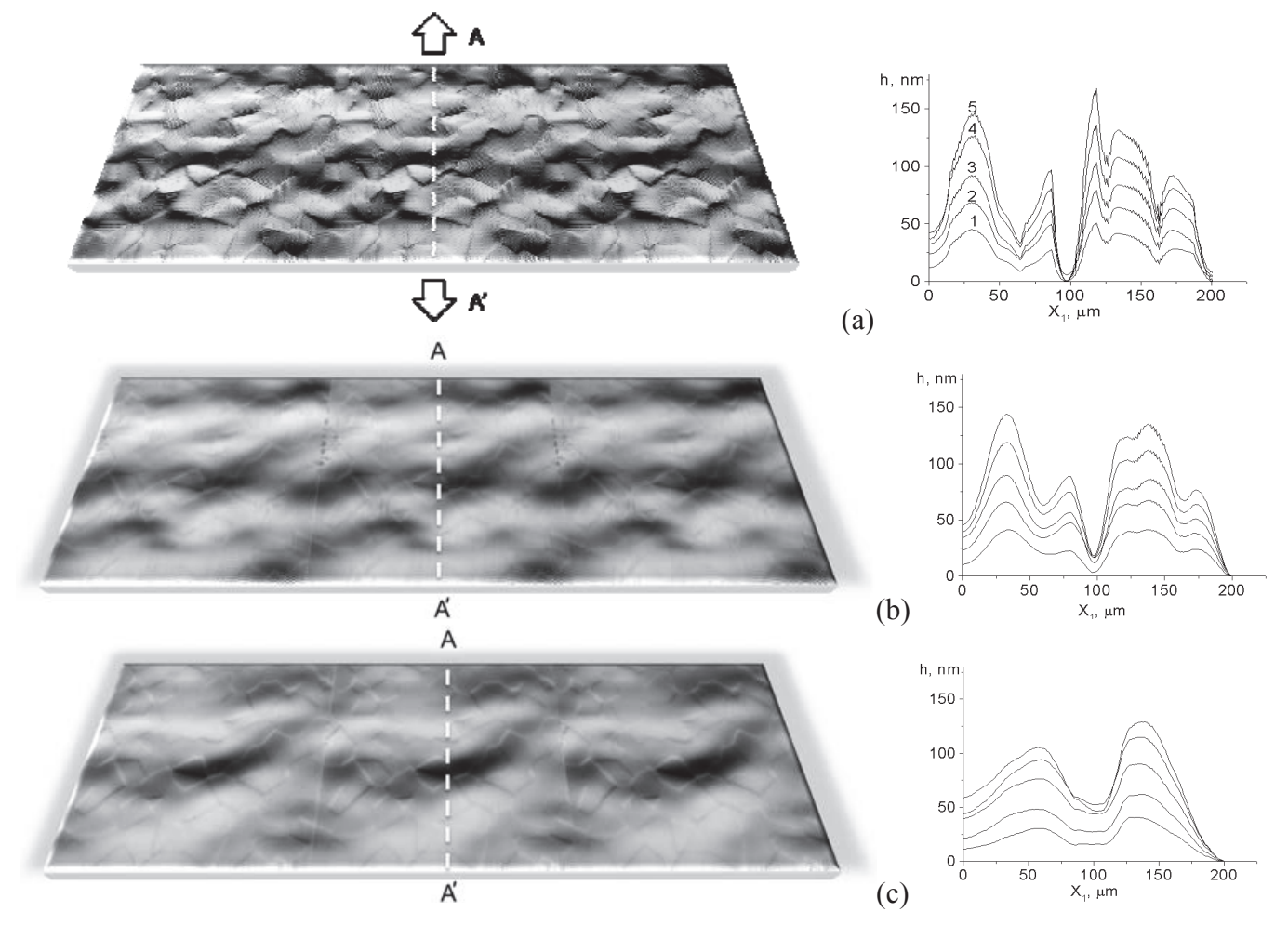

FIGURE 4. Surface roughness at a strain of $2.4 \%$ and corresponding surface profiles measured along $A-A^{\prime}$ lines in an unhardened specimen (a) and specimens with 10 (c) and $15 \mu \mathrm{m}$ (e) surface-hardened layers.

The numbers in subfigure $4 a$ correspond to $0.5,1,1.5,2$ and $2.4 \%$ of strains

Smaller folds evident in the structure of larger ones are associated with displacements of individual grains relative to each other. The surface-hardened layer possessing a quasi-homogeneous structure moves the sources of normal stresses (i.e. grain boundaries) away from the surface, thus suppressing the surface displacements. This conclusion is illustrated in Fig. 3 for the specimen with a $10 \mu \mathrm{m}$ thick surface-hardened layer within which the normal stresses are rather low (Fig. 3(a, b)). In the base material, however, the normal stresses amount to those observed in the unhardened specimen (cf. the stress profiles in Fig. 3(c)). Obviously, the thicker is the hardened layer, the lower are the normal stresses near the surface and the smoother is the surface relief (Fig. 4). The mesoscale fold width is much the same in all specimens, whereas the peak-to-valley height somewhat decreases as the surface-hardened layer grows thicker and fine surface folds caused by the motion of individual grains disappear. The latter effect is essential in view of the plastic strain localization which is much more pronounced in the case of the unhardened specimen due to steep gradients of the surface displacements.

Summarizing, we may conclude that an amorphous surface layer can smooth out surface roughening by suppressing low-scale displacements and thus diminishing the plastic strain localization on the specimen surface.

\section{ACKNOWLEDGMENTS}

This work is supported by Russian Science Foundation (grant No. 14-19-00766) and by a program to increase the competence of Tomsk Polytechnic University.

\section{REFERENCES}

1. D. Raabe, M. Sachtleber, H. Weiland, G. Scheele, and Z. Zhao, Acta Mater. 51(6), 1539 (2003).

2. M. R. Stoudt and J. B. Hubbard, Acta Mater. 53(16), 4293 (2005).

3. A. Panin, V. Romanova, R. Balokhonov, et al., Phys. Mesomech. 15(1-2), 94 (2012).

4. V. Romanova, R. Balokhonov, P. Makarov, et al., Comput. Mater. Sci. 28, 518 (2003). 